KODIFIZIERUNG DES VERWALTUNGSVERFAHRENSRECHTS: TRADITIONEN UND MODELLE

Der Beitrag erläutert die dem Verwaltungsverfahrensrecht zugrunde liegenden Gründe, Ziele und verfassungsrechtlichen Rahmenbedingungen. Darüber hinaus werden die Argumente für und gegen eine umfassende Kodifizierung von Verwaltungsverfahrensvorschriften sowie die wichtigsten Elemente des Verwaltungsverfahrensrechts erörtert. Zuletzt werden mögliche Kodifizierungsmodelle vorgestellt, nämlich das deutsche Verwaltungsverfahrensgesetz, das US-amerikanische Verwaltungsverfahrensgesetz und die ReNEUAL Modellregeln für das EU-Verwaltungsverfahren.

Schlüsselwörter: Verwaltungsrecht, gute Regierungsführung, Rechtsstaatlichkeit, Verwaltungsverfahrensregeln, Kodifizierung, Modelle, Deutsches Verwaltungsverfahrensgesetz, US-Verwaltungsverfahrensgesetz, NEUES Modell für EU-Verwaltungsverfahrensregeln. 


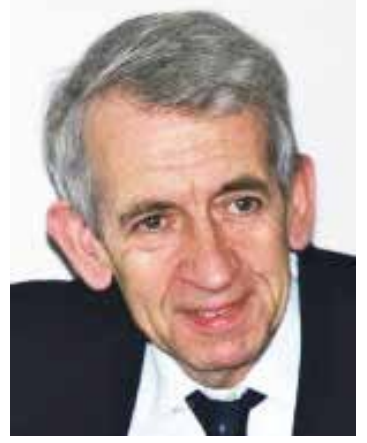

Professor Dr. Dres. h. c. Eberhard Schmidt-Aßmann, Professor (emeritus) des Öffentliches Rechts an der Universität Heidelberg e.schmidt-assmann@t-online.de
Das Verwaltungsverfahrensrecht ist ein zentraler Bestandteil jedes demokratischen und rechtsstaatlichen Verwaltungsrechts (Fromont, 2006: 209-231; Battini et al., 2007; Schneider, 2007; Schneider, 2009; Rose-Ackerman, Lindseth, 2010). Es sichert die Rechte der Bürger und vermittelt der Verwaltung Rationalität und Akzeptanz. Eine zunehmende Prozeduralisierung des Verwaltungsrechts ist, wie auch der Rechtsvergleich zeigt, eine allgemeine Entwicklungstendenz. „Die Notwendigkeit des Gesprächs zwischen Verwaltung und Bürger entspricht dem grundgesetzlichen Verständnis der Stellung des Bürgers im Staat" (Federal Constitutional Court, 1977: 297). Die Maßstäbe für „gute Verwaltung“ (good governance) sind zu einem erheblichen Teil Verwaltungsverfahrensrecht ${ }^{1}$. Das Konzept des modernen Verwaltungsrechts basiert auf Kommunikation und nicht auf Konfrontation (Pitschas, 1990).

Die folgenden Ausführungen beschäftigen sich zunächst allgemein mit den Zwecken und den verfassungsrechtlichen Grundlagen des Verwaltungsverfahrensrechts (abschnitt 1). Danach soll diskutiert werden, ob es sinnvoll ist, das Verwaltungsverfahren umfassend gesetzlich zu regeln (abschnitt 2) und welche „Modelle“ für eine solche Kodifikation zur Verfügung stehen (abschnitt 3).

1. Die "Idee“ des Verwaltungsverfahrens und die Grundlagen des Verwaltungsverfahrensrechts

Verfahren sollen in die Aktivitäten der Verwaltung Ordnung bringen: Die einzelnen Phasen des Entscheidens, die Rollen der daran Beteiligten, der Einfluss unterschiedlicher Interessen und die Bedeutung des Entscheidungsergebnisses sollen transparenter werden. Verfahren sind einerseits reale Abläufe und andererseits Ordnungsmuster (SchmidtAßmann, 2004: 46; Schmidt-Aßmann, 2012a). Sie beeinflussen die Anwendung des materiellen Rechts durch die Verwaltung und helfen, die Legalität der Verwaltung zu sichern.

Diese Einsicht ist nicht neu. Rechtliche Regeln über das Verfahren, das staatliche Amtsträger einhalten müssen, wenn sie Entscheidungen treffen wollen, entsprechen einer langen Rechtstradition. Ihre Grundelemente sind das Anhörungsrecht und das Neutralitätsgebot (Craig, 2012). Diese beiden Komponenten finden sich bereits im römischen Recht: „audiatur et altera pars" und „nemo iudex in causa sua“. In Deutschland

${ }^{1}$ Vgl. Art. 41 der Grundrechtecharta der Europäischen Union vom 12.12.2007. 
werden sie als Elemente des Rechtsstaatsprinzips angesehen. Andere Länder zählen sie zu den Allgemeinen Rechtsgrundsätzen. In England gehören sie zur "natural justice" und "procedural fairness". Mit Blick auf die Vereinigten Staaten von Amerika heißt es: "Natural justice plays much the same part in British law as does "due process of law" in the Constitution of the United States" (Wade, Forsyth, 2014).

\subsection{Plädoyer für einen weiten Begriff des Verwaltungsverfahrens}

Anhörungsrecht und Neutralitätsgebot zeigen, dass das Verfahren der Verwaltungsbehörden und das Verfahren der Gerichte gemeinsame Wurzeln haben. Das ist historisch erklärlich: In vielen Staaten hat sich die Verwaltung seit dem Mittelalter aus der Gerichtshoheit des Monarchen nach und nach ausdifferenziert. Auf diese Weise ist der Anwendungsbereich gerichtlicher Verfahrensregeln in das sich langsam entwickelnde Verwaltungsrecht transformiert worden.

Heute stellt sich die Situation jedoch differenzierter dar: Während das gerichtliche Verfahren einen relativ homogenen Typus bildet, in dem es um den Schutz individueller Rechte geht, sind die Aufgaben moderner Verwaltung so umfangreich und so vielgestaltig, das mehrere Verfahrenstypen herausgearbeitet werden müssen. Genannt seien beispielhaft:

- Verfahren zum Erlass von Einzelakten: dieses ist der „klassische“ Verfahrenstyp (z.B. Erlass von Steuerbescheiden, Verfügungen der Polizeibehörden, Genehmigung eines Baus oder einer gewerblichen Tätigkeit).

- Verfahren zum Abschluss von Verträgen der Verwaltung (z.B. Kauf oder Verkauf von Grundstücken, Vergabe öffentlicher Aufträge).

- Verfahren zur Aufstellung von Plänen (Straßenplanung, Stadtplanung, Planung von Naturschutzgebieten).

- Verfahren zum Erlass von Rechtsvorschriften der Verwaltung (Rechtsverordnungen, Satzungen der Gemeinden).

- Verfahren inter-behördlicher Kommunikation (z.B. Amtshilfeverfahren).

In der juristischen Literatur werden als weitere Typen beispielsweise genannt: Verteilungsverfahren, Überwachungsverfahren, Verfahren der Wissensgenerierung, Mediationsverfahren (Röhl, 2012). Alle diese Typen haben ihre eigenen Strukturen: Der Ablauf der Verfahren, die Konflikte, die sie bewältigen sollen, und die Verfahrensergebnisse sind ganz unterschiedlich. „Das“ Verwaltungsverfahren ist ein hoch abstrakter Begriff, der in einem modernen Verwaltungsrecht aber trotzdem seinen festen Platz haben muss. Das Verwaltungsverfahren ist neben den Handlungsformen der Verwaltung, den Verwaltungsrechtsverhältnissen und den Maßstäben des Verwaltungshandelns eines der vier Komponenten, nach denen sich die unüberschaubare Vielfalt der faktischen Verwaltungsaktivitäten rechtsstaatlich ordnen lässt (Schmidt-Aßmann, 2004: 32).

Die Feststellung, es gebe unterschiedliche Typen von Verfahren, heißt nicht, dass man alle diese Typen gesetzlich regeln sollte. Mein Plädoyer für einen weiten Begriff des Verwaltungsverfahrens dient zunächst nur dazu, sich der Weite des Themas bewusst zu sein. Rechtspolitische Diskussionen verengen sich oft viel zu schnell auf bestimmte Verfahrenstypen oder überhaupt nur auf einen einzigen Typ. Das ist ein Fehler. Zunächst muss es darum gehen, die Vielfalt unterschiedlicher Verfahren zu analysieren, vor allem die unterschiedlichen Interessen herauszuarbeiten, die in den Verfahren eine 
Rolle spielen. Ein Kapitel „Verfahren“ ist daher Bestandteil nahezu jedes Lehrbuchs zum Verwaltungsrecht.

Wenn das Verwaltungsverfahren als „Ordnungsidee“ dazu dienen soll, die Rationalität der Verwaltung zu verbessern, dann muss man das breite Spektrum der Verwaltungsaufgaben betrachten und überlegen, wie die Erfüllung der unterschiedlichen Aufgaben prozedural strukturiert werden kann. Hier sind die Wissenschaftlerinnen und Wissenschaftler an den Universitäten und Forschungsinstituten besonders herausgefordert. Sie sollten in der Lage sein, dem Gesetzgeber und der Verwaltung „Optionen“ zu benennen, wie diese Durchdringung des Verwaltungsalltags gelingen kann.

\subsection{Fragen der, Verfahrensphilosophie“6}

Welchen Stellenwert sollen Verwaltungsverfahren in der Rechtsordnung eines Landes haben? Die Antwort auf diese Frage ist teils rechtsdogmatischer und teils rechtspolitischer Art. Nicht selten hört man den Einwand, Verfahrensrecht schnüre die Verwaltung ein und mache sie unflexibel. Das wird vor allem von Praktikern in den Verwaltungen gern behauptet. Diese Behauptung ist nicht gänzlich falsch. Sie wird auch in Ländern diskutiert, in denen "procedural fairness" einen hohen Stellenwert besitzt (Schwartz, 1991). Natürlich kostet die Beachtung aller Verfahrensvorschriften Zeit. Es überwiegen jedoch die positiven Effekte: eine Steigerung der Qualität und eine höhere Akzeptanz der Verwaltungsentscheidungen. Die Prozeduralisierung des Verwaltungshandelns ist eine Frage des richtigen Maßes.

\subsection{1. „Doppelauftrag des Verwaltungsrechts“}

Verwaltungsverfahrensrecht ist von hoher Bedeutung. Aber man darf nicht in eine „Verfahrenseuphorie“ verfallen. Eine Verwaltungsentscheidung ist nicht schon deshalb besser, weil sie möglichst viele Verfahrensregeln einhalten muss. Dabei ist daran zu erinnern, dass das gesamte Verwaltungsrecht zwei Funktionen hat, die balanciert werden müssen:

- einerseits die Rechte des einzelnen Bürgers zu sichern (,Sicherungsauftrag“);

- andererseits die Verwaltung in die Lage zu versetzen, ihre Aufgaben im Rahmen des geltenden Rechts effektiv (,Effektuierungsauftrag“) zu erfüllen (Schmidt-Aßmann, 2004: 30).

Man kann das den „Doppelauftrag des Verwaltungsrechts“ nennen. In der älteren Theorie wurde oft allein der Sicherungsauftrag betont. Das ist jedoch zu einseitig. Die Verwaltung nimmt viele Aufgaben wahr, die für die Gesellschaft unverzichtbar sind. Sowenig das Verwaltungsrecht eines demokratischen Rechtsstaates einseitig auf die Interessen der Verwaltung ausgerichtet sein darf, sowenig darf es andererseits so konzipiert werden, dass es die Aktivitäten der Verwaltung permanent behindert.

Besonders einsichtig ist diese Erkenntnis dort, wo an einem Verwaltungsvorgang mehrere Bürger mit konträren Interessen beteiligt sind, also bei „mehrpoligen Rechtsverhältnissen“. Beispiel: Wer eine Fabrik bauen will, ist daran interessiert, die erforderliche Genehmigung möglichst schnell zu erhalten. Nachbarn und Umweltverbände heben dagegen die Nachteile hervor, die das Projekt für ihre Rechte und Interessen haben kann. Sie sind daran interessiert, dass die Verwaltung im Genehmigungsverfahren Schritt für Schritt, möglichst sorgfältig und das heißt im Regelfall auch langsam vorgeht. Dazu werden unter Umständen auch fern liegende Gefahren an die Wand gemalt und der Ruf 
nach immer neuen Gutachten erhoben. Soweit diese Maßnahmen notwendig sind, um eine in der Sache richtige Entscheidung treffen zu können, muss die Verwaltung diese Verfahrensschritte gehen. Auch hier kommt es aber auf das richtige Maß an. Dabei spielen die Pflicht der Verwaltung, den Sachverhalt vollständig aufzuklären, und die Beweislast eine Rolle. Gegebenenfalls muss die Verwaltung den Beteiligten eine Frist setzen, um ihre Einwände vorzutragen und nicht fristgerecht vorgetragene Einwände ausschließen (Institut der Präklusion). Das Beispiel zeigt: Verfahren sind notwendige Bedingungen sorgfältigen Entscheidens, aber sie sind keine „Verhinderungsstrategien“. Die Verwaltung und im Streitfall die Gerichte müssen jedes Verwaltungsverfahren so handhaben, dass der Doppelauftrag des Verwaltungsrechts erfüllt wird.

\subsubsection{Der Topos der „dienenden Funktion“ des Verfahrensrechts}

In diesen Zusammenhang gehört auch der Topos von der ,dienenden Funktion“ des Verwaltungsverfahrens. Er betrifft das Verhältnis des Verfahrensrechts zum materiellen Recht, also zu den sachlichen Vorgaben, die die einzelnen Vorschriften des Gewerberechts, des Steuerrechts oder Umweltrechts der Entscheidung der Verwaltung machen (Quabeck, 2010). Die Formel „dienende Funktion“ will ausdrücken, dass das Verfahrensrecht nicht so wichtig ist und es für die Entscheidungen der Verwaltung vor allem darauf ankommt, dass sie dem materiellen Recht entsprechen. Das klingt verfahrensökonomisch plausibel, führt aber zu einer Relativierung des Verfahrensrechts. Darin liegt eine erhebliche Gefahr für die Legalität der Verwaltung, denn die Verwaltung ist umfassend an das Recht und damit auch an das Verfahrensrecht gebunden.

Hier wird auch etwas von der „Verfahrensphilosophie“ deutlich, die in den einzelnen Staaten unterschiedlich ist. Eine sehr pauschale Einteilung sagt, dass in Deutschland traditionell der Vorrang des materiellen Rechts betont und damit dem Topos von der nur dienenden Funktion des Verfahrensrechts Raum gegeben werde, während England und die USA vom selbständigen Wert des Verfahrensrechts ausgehen würden. Das ist, wie gesagt, eine pauschale Aussage. Auch im englischen Recht findet sich der Topos der "serving function". Umgekehrt gibt es in Deutschland weite Bereiche des Verwaltungsrechts, in denen das Verfahrensrecht eine selbständige Funktion besitzt, vor allem überall dort, wo die Verwaltung nach Ermessen entscheiden muss, weil keine ganz exakten Vorgaben des materiellen Rechts existieren. Treffender als solche pauschalen Vergleiche lässt sich sagen: Die dienende Funktion des Verfahrensrechts ist ein nicht schlechthin unzulässiger Topos. Inwieweit von einer solchen Funktion auszugehen ist, hängt (erstens) davon $\mathrm{ab}$, welchen Zweck die einzelnen Verfahrensbestimmungen haben und (zweitens) wie genau das materielle Recht die Verwaltung programmiert. Verfahrensrecht ist also teils dienend, teils eigenständig gegenüber dem materiellen Recht (Pünder, 2014: 239). Wenn sich danach nicht feststellen lässt, dass das Verfahrensrecht in einem bestimmten gesetzlichen Kontext eine nur dienende Funktion haben soll, bleibt es bei der Grundregel, dass eine Verwaltungsentscheidung nur dann im vollen Sinne rechtmäßig ist, wenn sie dem materiellen und dem prozeduralen Recht entspricht.

\subsection{Die verfassungsrechtlichen Grundlagen des Verfahrensrechts}

Gibt es für das Verwaltungsverfahrensrecht Grundlagen in der Verfassung? Diese Frage ist nicht nur theoretisch, sondern auch praktisch wichtig. Soweit solche Grundlagen existieren, müssen sie bei der Schaffung und der Anwendung des Verfahrensrechts 
berücksichtigt werden. Die Verfassung des Staates ist kein bloßes politisches Programm, sondern sie ist Recht und hat folglich einen normativen Gehalt. Sie steht in ihrem Rang über dem ,einfachen Recht“, das heißt über den Gesetzen der Parlamente und den Verordnungen der Regierung. Diese sind an die Verfassung gebunden. Sie dürfen nicht gegen die Verfassung verstoßen und sind „verfassungskonform“ $\mathrm{zu}$ interpretieren.

\subsubsection{Die Bedeutung von Grundrechten}

Das gilt auch für das Verwaltungsverfahrensrecht. Soweit verfassungsrechtliche Grundlagen existieren, sind sie für das Parlament, wenn es Fragen des Verwaltungsverfahrens regelt, und vor allem für die Praxis der Verwaltung und der Gerichte unmittelbar relevant. So wird in den USA beispielsweise ausführlich erörtert, welche Regeln des Verwaltungsverfahrens Bestandteile der „due process clause“ des V. und des XIV. Amendments zur US-Verfassung von 1797 sind und welche es nicht sind (Schwartz, 1991). Nicht alle Verfahrensregelungen lassen sich auf die Verfassung zurückführen. Aber vor allem die Kernbestandteile eines Anhörungsrechts werden verfassungsunmittelbar durch die due process clause gewährleistet: "Before an agency takes action that adversely affects particular rights and obligations, those affected must be given to present their side of the case in a full and fair hearing" (Schwartz, 1991). Dazu zählen auch das Recht, eigene Beweise vorzulegen, die Unterlagen der Behörde zu kennen und am Ende eine Begründung des Verwaltungsakts zu erhalten, die darlegt, von welchen Tatsachen und Gründen die Behörde ausgeht.

In Deutschland spielen in diesem Zusammenhang die Grundrechte der Verfassung eine wichtige Rolle. „Grundrechtsschutz durch Verfahren“ ist ein zentral wichtiges Thema in Rechtsprechung und Literatur zum Verwaltungsverfahrensrecht (SchmidtAßmann, 2006). Das Verfahrensrecht wirkt als Verstärkung der materiellen Grundrechtsgehalte. Auch wenn der Text der einzelnen Garantienorm darüber nichts aussagt, ist die prozedurale Dimension mit im Blick zu behalten.

Leitfall ist die „Mülheim-Kärlich“-Entscheidung des Bundesverfassungsgerichts (1. Senat) aus dem Jahre 1979 (Federal Constitutional Court, 1979: 30). Der Rechtsstreit betraf die Genehmigung eines Atomkraftwerkes: konkret die Klage einer Nachbarin gegen die Genehmigung und gegen das für die Genehmigung notwendige Verwaltungsverfahren. Das Gericht stellt zunächst heraus, dass sich die Klägerin angesichts des Gefahrenpotentials von Atomkraftwerken auf das Grundrecht auf Leben und körperliche Unversehrtheit (Art. 2 Abs. 2 Grundgesetz) berufen könne (Federal Constitutional Court, 1979: 57). Dem Schutz dieser Rechtsgüter diene - so folgert das Gericht weiter - das gesetzliche Erfordernis einer Genehmigung und das bei der Erteilung dieser Genehmigung einzuhaltende Verwaltungsverfahren. Das Grundrecht strahlt auf die Gestaltung und die Anwendung des Verfahrensrechts aus. Im Streitfall konnte die Mehrheit des 1. Senats einen Verstoß gegen das einschlägige Verfahrensrecht nicht feststellen, so dass die Klägerin unterlag. Das Minderheitsvotum der Verfassungsrichter Simon und Heußner stellt die Bedeutung von Verwaltungsverfahren für den Schutz der Grundrechte noch deutlicher heraus und rügt die Art, in der die Behörden das streitige Verfahren geführt haben: „Das Ausgangsverfahren ist geradezu ein Lehrstück für eine Verfahrensgestaltung, die zwar die reibungslose Durchführung des behördlichen Verfahrens erleichtert, die aber die Mitwirkungsrechte betroffener Bürger überspielt und 
deren ohnehin vorhandene Ohnmachtserfahrungen gegenüber dem Staatsapparat und einflussreichen Interessenten bestätigt“ (Federal Constitutional Court, 1979: 70).

Jedenfalls sind im Gefolge dieser Judikatur des Bundesverfassungsgerichts Gesetzgebung, Verwaltung und Gerichte wesentlich sensibler für die verfassungsrechtlichen Dimensionen des Verwaltungsverfahrens geworden. Die Grundrechte, die Gewährleistung umfassenden Gerichtsschutzes (Art. 19 Abs. 4 Grundgesetz) und das Rechtsstaatsprinzip haben unmittelbare Bedeutung für die gesetzgeberische Ausgestaltung des Verwaltungsverfahrens und für die Anwendung vorhandenen Verfahrensrechts durch die Verwaltung und die Gerichte. In diesem Sinne haben das Anhörungsrecht der von einer Verwaltungsentscheidung betroffenen Bürger und das Neutralitätsgebot Verfassungsrang. Ihre Ausgestaltung im Einzelnen darf der Gesetzgeber nach seinen eigenen Vorstellungen vornehmen. Er besitzt dabei einen erheblichen Gestaltungsspielraum. Aber er muss immer die verfassungsrechtliche Basis im Auge behalten. Eine Ausgestaltung, die die Ausübung dieser Rechte praktisch unmöglich machen oder unzumutbar erschweren würde, ist verfassungsrechtlich unzulässig.

\subsubsection{Die Bedeutung des Demokratieprinzips}

Dass auch das demokratische Prinzip der Verfassungen die Ausgestaltung des Verwaltungsverfahrens beeinflusst, ist heute ebenfalls anerkannt. Der einschlägige Topos ist die „Partizipation“. Allerdings sind die Zusammenhänge hier nicht so eindeutig wie bei den Grundrechten und dem Rechtsstaatsprinzip. Jedenfalls zeigt der Rechtsvergleich größere Unterschiede:

- In den USA wird häufig auf das Demokratieprinzip zurückgegriffen, wenn es um Fragen des Verwaltungsverfahrens geht. Die Argumentation ist etwa die folgende: Die demokratische Legitimation der Verwaltung durch die parlamentarische Gesetzgebung sei ungenügend, weil die Gesetze sehr oft viel politische Macht delegieren würden (Rose-Ackerman, Perroud, 2013: 225). Die Ausübung dieser Macht müsse dann dadurch begrenzt werden, dass betroffene und interessierte Bürger und Interessengruppen in breitem Umfang die Möglichkeit erhalten, der Verwaltung ihre Ansichten zur Kenntnis zu geben. Die Verwaltung muss sich dann mit diesen Ansichten intensiv auseinandersetzen, bevor sie ihre Entscheidung trifft. Das Verwaltungsverfahren entfaltet hier im Rahmen des Demokratieprinzips eine Art Kompensationsfunktion. Auf dieser Vorstellung basiert auch der Administrative Procedure Act der USA aus dem Jahre 1946.

- Auch in Deutschland ist anerkannt, dass das Demokratieprinzip der Verfassung (Art. 20 und 28 Grundgesetz) für Verwaltungsverfahren Bedeutung hat (Trute, 2012). Das gilt etwa für die Verfahren der kommunalen und funktionalen Selbstverwaltung. Grundsätzlich geht man jedoch davon aus, dass die demokratische Legitimation der Verwaltung sich primär auf dem Weg über das Parlament vollzieht: Steuerung durch das parlamentarische Gesetz und den Haushalt (sachliche Legitimation), parlamentarisch eingesetzte und kontrollierte Regierung und Verwaltung (organisatorische Legitimation) (Federal Constitutional Court, 1990: 37, 60). Inwieweit daneben eine Partizipation von Betroffenen, Interessengruppe und Verbänden im Verwaltungsverfahren die demokratische Legitimation der Verwaltung erhöht, wird unterschiedlich bewertet. Die ältere Lehre betont eher, dass die Legitimation der Verwaltung seitens des Parlaments auf diese Weise beeinträchtigt werden kann, weil die Verwaltung dadurch schwer zu 
durchschauenden Einflüssen besonders aktiver Lobbygruppen ausgesetzt wird. Eine neuere Ansicht in der Literatur bewertet die demokratische Bedeutung der Partizipation im Verwaltungsverfahren demgegenüber positiv (Trute, 2012). Auch diese Ansicht betont allerdings, dass die mit der Partizipation eröffneten Einflussmöglichkeiten rechtlich geordnet und transparent gestaltet werden müssen. Andernfalls kann Partizipation die Gleichheit der Beteiligungschancen, die die Demokratie allen Bürgern gewährt, verletzt werden. Keinesfalls darf ein Verwaltungsverfahren so geführt werden, dass die zuständige Verwaltung ihre Entscheidung an Gremien oder Interessengruppen, die ihrerseits nicht demokratisch legitimiert sind, praktisch delegiert.

\subsubsection{Unterschiedliche Konsequenzen für unterschiedliche Typen von Verfahren}

Als eine allgemeine Erkenntnis zur Frage der Verfassungsrelevanz des Verwaltungsverfahrens kann festgehalten werden: Die Art und die Intensität der verfassungsrechtlichen Einflüsse hängen sehr von dem Typ des Verwaltungsverfahren ab, um das es geht. Oben wurde schon verdeutlicht, dass es nicht „das“ Verwaltungsverfahren gibt, sondern verschiedene Typen, die sich in ihren Aufgaben, in ihren Strukturen und in ihren Ergebnissen wesentlich unterscheiden. Das ist auch bei der Frage der Verfassungsrelevanz zu beachten: Für Verfahren zu Erlass von Einzelakten stehen die individuellen Rechte der Betroffenen im Zentrum. Folglich sind hier vor allem die Grundrechte als verfassungsrechtlicher Rahmen wichtig. Geht es dagegen um Planungs- und Normsetzungsverfahren, so sind Einflüsse des demokratischen Prinzips zu bedenken. Für Verfahren der Sozialverwaltung kann das Sozialstaatsprinzip Bedeutung erlangen und zum Beispiel Pflichten der Verwaltung begründen, sozial schwache Bürger besonders sorgfältig über ihre Rechte zu beraten. Wenn die Verfassung ein Staatsziel „Umweltschutz“ festlegt, kann es geboten sein, dass umweltrechtliche Vorsorgeprinzip prozedural besonders abzusichern, z.B. durch besondere Verwaltungsverfahren der Risikoabschätzung und des Risikomanagements (Jaeckel, 2010: 220; Wollenschläger, 2009). Einheitlich für alle Verfahrenstypen gilt das Neutralitätsgebot. Es ist im Rechtsstaatsprinzip und auch im Demokratieprinzip verankert.

\section{Die Kodifikation von Verwaltungsverfahrensrecht}

Verwaltungsverfahrensrecht findet sich üblicherweise an ganz unterschiedlichen Stellen der Rechtsordnung geregelt:

- Fachgesetze: Oft enthalten schon die Gesetze, die einzelne Aufgaben der Verwaltung regeln (also die Steuergesetze, Polizeigesetze oder Umweltgesetze), auch Regelungen zu einzelnen Verfahrensfragen: z.B. Vorschriften über die Zuständigkeiten oder über die Befugnis zu Inspektionen und anderen Aufklärungsmaßnahmen. Doch sind diese Vorschriften in ihrem Anwendungsbereich auf das jeweilige Gesetz beschränkt und erfassen die Verfahrensprobleme nur punktuell.

- Teil-Kodifikationen: In manchen Ländern sind durch Gesetze aber auch einzelne größere zusammenhängende Verfahrensprobleme geregelt worden. Ein schon älteres Beispiel bildet das Vollstreckungsrecht der Verwaltung. In jüngerer Zeit sind es die Datenschutzgesetze und die Informationsfreiheitsgesetze, die Verfahrensrecht für die Verwaltung geschaffen haben. Manche Länder haben das Recht das Vergaberecht der Verwaltung (public procurement) in einem eigenen Gesetz geregelt und damit eine „Querschnittregelung“ geschaffen, die überall greift, wo die Verwaltung Waren oder Dienstleistungen auf dem freien Markt „einkaufen“ will. 
- Richterrecht: Als klassische Rechtsquelle des Verwaltungsverfahrensrechts gilt das Richterrecht. In historischer Perspektive lässt sich sogar sagen: die meisten elementaren Kenntnisse von dem, was die Verwaltung bei ihrem Vorgehen zu beachten hat, verdanken wir den Gerichten. Die Gerichte haben sie „Schritt für Schritt“ entwickelt, verdichtet und gegebenenfalls auch umgestaltet.

\subsection{Vorzüge und Nachteile von Kodifikationen}

Was kann eine „Kodifikation“ des Verwaltungsverfahrensrechts angesichts dieser Situation bedeuten?

Zunächst einmal zum Begriff: Eine Kodifikation ist eine nach übergreifenden Prinzipien getroffene Regelung eines größeren Rechtsgebietes. Ihr Kennzeichen ist systematische Geschlossenheit. Die bloße Zusammenfassung ganz unterschiedlicher Regelungen in einem einheitlichen Rechtsakt sollte nicht als Kodifikation bezeichnet werden. Einer Kodifikation geht es darum, die erfassten Teile inhaltlich auf gewisse einheitliche Linien, auf „Systemgedanken“, auszurichten. Das setzt ein bestimmtes Maß an „Kodifikationsreife" und außerdem eine gründliche Vorbereitung im politischen und im wissenschaftlichen Raum voraus. Wenn sich ein Rechtgebiet noch im Experimentierstadium befindet, ist es nicht sinnvoll, es schon zu kodifizieren. Vielfach erfolgen Kodifikationen durch parlamentarisches Gesetz. Notwendig ist das jedoch nicht. Die jüngst erfolgte Kodifikation des französischen Verwaltungsverfahrensrechts wurde durch einen Rechtsakt des Staatspräsidenten erlassen.

Kodifikationen sind rechtspolitisch keine Selbstläufer. Sie stoßen nicht selten auf erheblichen Widerstand. Das gilt gerade auch für Kodifikationen des Verwaltungsverfahrensrechts. Besonders starke Abneigungen gibt es in England. Befürchtet wird eine zu starke Einschnürung der Verwaltung in abstrakte Begriffe ${ }^{2}$. Die Fortentwicklung des Verwaltungsverfahrens durch die Gerichte erscheint als der flexiblere Weg. Dass diese Einschätzung allerdings nicht im gesamten angelsächsischen Kontext geteilt wird, zeigen die USA, die seit 1946 über einen Administrative Procedure Act verfügen ${ }^{3}$.

In Deutschland hat der Kodifikationsgedanke einen positiven Klang. Trotzdem gehörte Deutschland, was das Verwaltungsrecht anbetrifft, nicht zu den Vorreitern (Schneider, 2012). Als das Verwaltungsverfahrensgesetz 1976 erlassen wurde, verfügten etwa Spanien, Österreich und Polen längst über entsprechende Kodifikationen. Seither hat die Kodifikationsidee in Europa allerdings an Boden gewonnen (Schneider et al., 2015). $\mathrm{Zu}$ nennen sind etwa Italien und die Niederlande und seit Oktober 2015 auch Frankreich, das bisher zu den Gegnern einer Kodifikation des Verwaltungsverfahrens gehörte. Selbst auf der Ebene der Europäischen Union, die sonst durch eine starke Fragmentierung ihrer Politik gekennzeichnet ist, zeigen sich neuerdings Kodifikationstendenzen, auf die ich später genauer eingehen werde (vgl. unter C III).

Welche Vorteile lassen sich für eine Kodifikation des Verwaltungsverfahrensrechts anführen? Diese Frage nach dem „Mehrwert“" einer Kodifikation ist bekannt (German Bundestag, 1973). Ich kann mich daher kurz fassen und dabei auf einige Konsequenzen hinweisen.

${ }^{2}$ Eine geschlossene Front der Ablehnung existiert freilich nicht (Craig, 2013: 503). Im Grunde herrscht in England insofern ,a mix of judge-made principles and sector-specific rules” (Bell, 2006: 1259).

${ }^{3}$ Dazu unten unter 3.2. 
(1) An erster Stelle dient eine Kodifikation des Verwaltungsverfahrensrechts der Information des Bürgers über seine verfahrensmäßigen Rechte (und eventuell Pflichten) gegenüber der Verwaltung. Dazu wurde bei der Vorbereitung des deutschen Verwaltungsverfahrensgesetztes etwa Folgendes gesagt (Schwartz, 1991): „Für den Staatsbürger bedeutet die Vereinheitlichung und Kodifizierung des Verwaltungsverfahrens eine Stärkung seiner Rechtsstellung. Für ihn ist von besonderer Bedeutung, dass ein einheitliches Verfahrensrecht die Tätigkeit der Behörden übersichtlicher und ihm verständlicher macht. Dadurch wird gleichzeitig auch dem Grundsatz der Rechtssicherheit Rechnung getragen“".

Selbst wenn die Bürger den Text der Kodifikation nicht täglich als Buch unter dem Arm tragen, ist allein schon das Bestehen eines solchen Codes hilfreich. Dazu ist es erforderlich, dass die Bürger von der Existenz erfahren (Schulbildung, Information auf Internetseiten). Die Verwaltung hat insofern eine politische Informationspflicht.

Die Informationsfunktion ist nur erfolgreich, wenn die Kodifikation klar gegliedert ist und ihr Umfang nicht ausufert. Mehrere hundert Einzelvorschriften sind eher hinderlich. Wichtig ist ferner eine einfache Sprache, die aber juristisch exakt sein muss. Es sollten schließlich nur solche Bestimmungen aufgenommen werden, die rechtlich bindend und durchsetzbar sind. Dem Bürger sollte „reiner Wein eingeschenkt“ werden, was er von der Verwaltung in verfahrensmäßiger Hinsicht von Rechts wegen verlangen kann. Ein Verfahrensgesetz sollte keine Lyrik enthalten.

(2) Für die in der Verwaltung tätigen Amtsträger stellt eine Kodifikation des Verwaltungsverfahrensrechts eine wichtige Grundlage ihrer täglichen Arbeit dar. Sie standardisiert die Antworten auf Rechtsfragen, die sich bei der Bearbeitung von Fällen stellen. Dadurch stützt sie die gleichmäßige Behandlung von Einzelfällen und wirkt willkürlichen Entscheidungen von Behörden entgegen. Dazu ist es notwendig, dass eine Kodifikation nicht nur allgemein gehaltene Grundsätze enthält. Notwendig sind vielmehr konkret gefasste Tatbestände, die die Verfahrensrechte und gegebenenfalls auch die zulässigen Ausnahmen festlegen. Das entlastet die Amtsträger im Kontakt mit dem Publikum. Wie der Bürger so können auch sie auf einen bindenden Rechtstext verweisen, von dem sie nicht abweichen dürfen. Verwaltungsverfahrensgesetze entfalten auf diese Weise eine Edukationsfunktion gerade dort, wo es darum geht, eine rechtsstaatliche Verwaltung aufzubauen.

(3) Für die Praxis der Gerichte haben Kodifikationen eine Kristallisationsfunktion. Weil Kodifikationen ,allgemeine“ Regelungen bieten, können die Gerichte diese Vorschriften als "Sammelpunkte“ auch für Urteile verwenden, die sich mit vergleichbaren Fragen aus ganz unterschiedlichen Rechtsgebieten beschäftigen. Beispiel: Wenn eine Kodifikation der Verwaltung allgemein Regelungen an die Hand gibt, wie sie ihr Ermessen auszuüben hat, werden auch diejenigen Urteile diese Regelungen reflektieren, wenn sie sich mit der Ermessensausübung in einem fremden Rechtsgebiet beschäftigen müssen.

Unterstützt wird diese Funktion dann, wenn Kommentare zum Verwaltungsverfahrensgesetz entstehen. Die Kodifikation wird auf diese Weise zur zentralen Referenz für die Dokumentation und die Fortentwicklung des Allgemeinen Verwaltungsrechts. Besonders günstig ist es, wenn die Kommentare als Gemeinschaftswerke von Richtern, Verwaltungsbeamten und Professoren konzipiert sind. Der Austausch zwischen Praxis und Wissenschaft wird auf diese Weise über die Kodifikation vermittelt. 
(4) Für die Gesetzgebung haben Kodifikationen eine Entlastungsfunktion. Was allgemein geregelt ist, muss nicht in jedem neuen Gesetz erneut festgelegt werden. Zugleich wird dadurch der Druck verringert, den Lobbygruppen auf den Gesetzgeber ausüben. Natürlich ist es zulässig, dass ein späteres Gesetz für einen bestimmten Bereich eine andere Regelung trifft, als sie in der Kodifikation getroffen ist. Rechtlich hat eine Kodifikation keinen höheren Rang als andere Gesetze. Aber es sollte sich nach und nach eine politische Regel entwickeln: Wenn ein jüngeres Gesetz eine Sonderregelung schaffen will, die von den Vorschriften der Kodifikation abweicht, dann müssen dafür tragfähige Gründe genannt werden. Man kann das die „disziplinierende Kraft" nennen, die eine allgemeine Regelung gegenüber Sonderwünschen entfaltet. Dass eine solche Kraft im politischen Raum nur langsam entwickelt werden kann und immer wieder mit Rückschlägen gerechnet werden muss, ist absehbar. Auch die Entwicklung des deutschen Verwaltungsverfahrensgesetzes ist nicht frei von solchen Rückschlägen. Die Literatur kritisiert zu Recht immer wieder Tendenzen der „De-kodifikation“, weil Politiker und einflussreiche Verbände versuchen, den erreichten Stand an Einheitlichkeit durch Sonderwünsche aufzubrechen (Kahl, 2002: 67).

Eine Kodifikation des Verwaltungsverfahrensrechts wirkt - im Guten wie im Schlechten - wie ein Spiegel der gegenwärtigen Verwaltungskultur eines Landes.

\subsection{Die wichtigsten Bauelemente eines Verwaltungsverfahrensrechts}

Nach diesen rechtspolitischen Überlegungen kehren wir $\mathrm{zu}$ rechtsdogmatischen Problemen zurück und geben einen Überblick über die wichtigsten Verfahrensregelungen, die sich aus der Rechtsvergleichung im Verwaltungsrecht gewinnen lassen. Die „Modelle“ von Kodifikationen, die später behandelt werden sollen (vgl. unter C), stellen Kombinationen unterschiedlicher „Bauformen“ dar. Sie stellen ,prozedurale Arrangements" zur Verfügung.

\subsubsection{Elemente eines "Standardverfahrens“}

Die folgenden Bauformen sind für ein rechtsstaatliches Verfahrensrecht schlechthin unverzichtbar". Sie bilden zusammen etwas, was man ein „Standardverfahren“ nennen kann (Schneider, 2012).

\subsubsection{Regelungen der Zuständigkeit}

Zunächst einmal muss die zentrale Frage geregelt sein, welche Behörde für den Ablauf und den Abschluss des Verfahrens verantwortlich ist.

Weiter gehören hierher auch Bestimmungen zum Neutralitätsgrundsatz (,nemo iudex in causa sua"): In welchen Fällen ist eine Behörde oder eine Beamter befangen, weil er durch Verwandtschaft oder Freundschaft ein persönliches Interesse an dem Verfahren hat? Wie wird diese Befangenheit festgestellt? Welche Konsequenzen hat es, wenn ein befangener Beamter an den Verfahren mitgewirkt hat?

2.2.1.2. Regelungen über die Beteiligten

Wer soll an dem Verfahren beteiligt sein? Auf jeden Fall ist das der Adressat der beabsichtigten Verwaltungsentscheidung, ferner derjenige, der das Verfahren beant-

${ }^{4}$ Vgl. Art. 41 der Grundrechtecharta der Europäischen Union; außerdem zahlreiche Empfehlungen des Europarates (Schmidt-Aßmann, 2012a). 
ragt hat (Antragsteller). In Betracht kommen auch Dritte, deren berechtigte Interessen in dem Verfahren mit behandelt werden oder durch das Ergebnis des Verfahrens beeinträchtigt werden können, z.B. Nachbarn oder Konkurrenten eines Gewerbetreibenden. Schließlich ist es denkbar, die Öffentlichkeit an einem Verfahren zu beteiligen (vgl. unter 2).

\subsubsection{Verfahrensrechte der Beteiligten}

Welche Rechte sollen die Beteiligten im Verwaltungsverfahren haben?

- Auf jeden Fall ein Recht auf Anhörung, bevor die Verwaltung eine belastende Entscheidung trifft. Dieses Recht folgt aus den Grund- und Menschenrechten und aus dem Gedanken der procedural fairness.

- Damit das Anhörungsrecht effektiv ausgeübt werden kann, sollte außerdem das Recht festgelegt werden, dass die Beteiligen die Akten der Behörde einsehen können. Geregelt werden sollte auch, in welchen Fällen sich die Behörde demgegenüber darauf berufen darf, dass die Akten geheim bleiben müssen.

In diesen Kontext gehört auch das Recht jedes Beteiligten, sich eines sachkundigen Beistandes zu bedienen; ferner die Pflicht der Behörde, die Beteiligten über ihre prozeduralen Rechte aufzuklären.

\subsubsection{Regelungen zum Beweisrecht}

Diese Regelungen legen fest, von wem und mit welchen Mitteln der Sachverhalt aufgeklärt werden muss.

- In vielen kontinental-europäischen Rechtsordnungen besteht für die Behörde die Pflicht, den Sachverhalt von Amts wegen aufzuklären. Die Beteiligten müssen daran „mitwirken“. Aber die wichtigsten Aufgaben weist der Untersuchungsgrundsatz der Behörde zu. Sie muss dabei auch Fakten aufklären, die gegen ihre eigene Ansichten und Vermutungen sprechen könnten.

- Welche Mittel darf die Behörde einsetzen, um den Sachverhalt aufzuklären, z.B. Zeugen vernehmen, Auskünfte bei anderen Behörden einholen? Soweit solche Maßmahnen mit Eingriffen in die Rechte der Bürger verbunden sind, muss dafür eine eigene gesetzliche Ermächtigung vorhanden sein, z.B. für Inspektionen oder für geheime Datenerhebungen mittels technischer Einrichtungen.

\subsubsection{Regelungen zum Abschluss des Verwaltungsverfahrens}

- Soll die Behörde verpflichtet sein, das Verfahren in einer gesetzlich bestimmten Frist abzuschließen?

- Wem und wie ist die Entscheidung den Beteiligten bekanntzugeben?

- In welcher Form ist das Verfahren abzuschließen; z.B. durch den Erlass einer förmlichen Entscheidung oder informell?

- Muss die Verwaltung ihre Entscheidung (schriftlich) begründen? Eine solche Begründungspflicht kennt das deutsche Recht und das Recht der Europäischen Union. In England ist eine Begründung erst notwendig, wenn es später zu einer Kontrolle der Entscheidung (judicial review) kommt (Craig, 2012).

- Muss die Verwaltung die Beteiligten darüber informieren, wie sie Rechtsschutz gegen die Entscheidung erlangen können? Eine solche Belehrungspflicht gehörte bisher nicht durchgängig zu den notwendigen Gewährleistungen. In neuerer Zeit aber wird eine solche Belehrung mehr und mehr verlangt (vgl. z.B. § 37 Absatz 6 VwVfG). 


\subsection{Verfahren unter Beteiligung der Öffentlichkeit}

Verfahren, an denen eine große Zahl von Personen oder überhaupt die Öffentlichkeit beteiligt ist, gab es vereinzelt schon früher, z.B. im Wasserrecht oder beim Bau von Straßen. In jüngerer Zeit haben diese Verfahren erheblich zugenommen. Vor allem das Umweltrecht der Europäischen Union verpflichtet die Mitgliedstaaten, immer dann, wenn es um umweltrelevante Planungen und Entscheidungen der Verwaltung geht, in einem förmlichen Verfahren die Öffentlichkeit zu beteiligen (Umweltverträglichkeitsprüfung).

Verfahren mit Öffentlichkeitsbeteiligung sind komplexe Verwaltungsverfahren, die detaillierte Vorschriften verlangen. Mindestens müssen Vorschriften zu folgenden Fragen geschaffen werden:

- Wer zählt zur Öffentlichkeit (nur Betroffene wie Nachbarn oder jedermann)?

- Auf welche Weise ist die Öffentlichkeit über den Gegenstand des Verfahrens zu informieren (öffentliche Auslegung von Akten, Zeitungen)?

- Wie kann die Öffentlichkeit sich zu dem Gegenstand äußern (mündlich, schriftlich, über das Internet)?

- Welche Bedeutung haben die von der Öffentlichkeit beigetragenen Informationen für die Entscheidung der Verwaltung?

Varianten dieses Verfahrens sind solche Verfahren, in denen ein großer Kreis von Betroffenen seine Argumente in einer mündlichen Verhandlung vortragen kann. Es kann sich auch um ein formalisiertes Hearing handeln, das ähnlich wie ein Gerichtsverfahren durchzuführen ist. Um die Neutralität der Verwaltung zu erhöhen, sollte in der Art einer „inneradministrativen Gewaltenteilung“ zwischen demjenigen Beamten, der das Hearing durchführt, und demjenigen, der die endgültige Entscheidung in der Sache trifft, getrennt werden.

\subsection{Weitere Gegenstände von Kodifikationen von Verwaltungsverfahren}

Es gibt viele weitere Bauformen und prozedurale Arrangements, die in eine Kodifikation des Verwaltungsverfahrens einbezogen werden können. Jede von ihnen besteht aus zahlreichen Einzelregelungen. Ob sie einbezogen werden sollen, ist eine Frage der Zweckmäßigkeit. Um die Transparenz der Kodifikation nicht zu gefährden, ist eine restriktive Linie zu empfehlen. In Betracht kommen:

- Regelungen der Verwaltungsvollstreckung: Das sind Fragen, die sich an ein durchgeführtes Verwaltungsverfahren anschließen. In der Sache besteht also ein plausibler Zusammenhang.

- Regelungen der Rechtsform der Verfahrensergebnisse, also z. B. über Verwaltungsakte oder über Verträge der Verwaltung: sog. konnexe Materien. Sie gehen über rein prozedurale Fragen hinaus, haben aber ebenfalls einen plausiblen Zusammenhang mit diesen.

- Regelungen zum Datenschutz und zur Frage eines allgemeinen Zugangs zu den Akten der Verwaltung: Diese beiden Kernfragen des ,Informationsverwaltungsrechts “ betreffen ein Thema, das sich nicht nur in einem laufenden Verfahren, sondern allgemein stellt. Für die Verwaltungskultur ist es ein besonders wichtiges Thema. Das legt es nahe, solche Regelungen in ein Verwaltungsverfahrensgesetz einzubeziehen. Zwingend ist das aber nicht.

- Regelungen der Amtshilfe und des Informationsaustausches zwischen Behörden. 


\section{Drei Beispiele für Kodifikationen als „Modelle“6}

Die voraufgehenden Untersuchungen haben schon gezeigt, wie unterschiedlich Kodifikationen des Verwaltungsverfahrensrechts gestaltet sein können. In der Praxis hängt Vieles von der historischen und der politischen Situation ab, in der eine Kodifikation entstanden ist (Deppe, Pudelka, 2014). Aber es wird auch deutlich, dass der Vorgang der Kodifikation meistens gut genutzt worden ist, um den Zustand des Verwaltungsverfahrens im eigenen Lande gründlich zu reflektieren und für die Zukunft Perspektiven zu entwickeln. Kodifikationsprozesse sind Lernprozesse. Sie stärken das konzeptionelle Denken und sind daher gerade in Zeiten, die in vielen Politikbereichen von einem „,muddling through" beherrscht sind, ein wichtiges Gegengewicht.

Im Folgenden sollen drei Kodifikationen vorgestellt werden, an denen sich die Vorzüge konzeptionellen Denkens zeigen: das deutsche Verwaltungsverfahrensgesetz von 1976 (VwVfG), der US-amerikanische Administrative Procedure Act von 1946 (APA) und der ReNEUAL-Musterentwurf für ein EU-Verwaltungsverfahrensrecht von 2014.

\subsection{Das deutsche Verwaltungsverfahrensgesetz (VwVfG) von 1976}

Deutschland ist ein Bundesstaat. Nach dem deutschen föderalen System ist der Vollzug von Gesetzen grundsätzlich Sache der Länder. Der Bund nimmt nur verhältnismäßig wenige Verwaltungsaufgaben wahr (allerdings mit wachsender Tendenz). Die Verwaltungen der Länder und der Gemeinden vollziehen nicht nur das Landesrecht, sondern auch das Bundesrecht. Folglich finden sich die ersten Initiativen, das Verwaltungsverfahrensrecht zu kodifizieren, auf der Ebene der Länder. Diese Initiativen reichen teilweise in die Zeit vor dem 2. Weltkrieg zurück (Schmidt-Aßmann, 2012a; Schneider, 2012). Ab 1955 wurde immer mehr diskutiert, ob es nicht notwendig sei, ein für die Verwaltungen der Länder und des Bundes möglichst einheitliches Verwaltungsverfahrensrecht zu schaffen. Dabei fiel dem Bund die Rolle eines Vorreiters zu.

Der Kodifikationsprozess dauerte 20 Jahre. An den Diskussionen waren nicht nur die zuständigen Ministerien sondern auch Richter, Professoren, Rechtsanwälte und Einrichtungen der Zivilgesellschaft beteiligt. Zunächst gab es erheblichen Widerstand gegen ein solches Projekt. Befürchtet wurde eine „Einschnürung“ der Verwaltung; das bisherige Richterrecht sei, so sagte man, flexibler. Es waren etwa dieselben Argumente, wie sie heute noch die englische Diskussion beherrschen. Endlich aber siegten die Befürworter. Heute sind diese Diskussionen verstummt. Natürlich gibt es Kritik, nicht nur an einzelnen Vorschriften des VwVfG. Aber zurück zu einer kodifikationslosen Zeit will niemand. Die Kodifikation hat im deutschen Verwaltungsrecht heute seinen zentralen und festen Platz.

\subsubsection{Das Konzept}

Das VwVfG ist das Verfahrensgesetz der klassischen gesetzesvollziehenden Verwaltung (Schmidt-Aßmann, 2012a). Es rückt das Verhältnis des Einzelnen zur Verwaltung in den Mittelpunkt. Dieses Verhältnis soll rechtsstaatlich geregelt werden. Dazu wird der Einzelne mit einer Reihe von Verfahrensrechten ausgestattet und die Verwaltung mit zahlreichen Pflichten belegt. Die meisten von ihnen lassen sich auf die Grundgedanken des Anhörungsrechts und der administrativen Neutralitätspflicht zurückführen (vgl. oben A I). Erfasst werden vor allem Verwaltungsvorgänge „,vor Ort“, die im täglichen Leben 
der Bürger eine Rolle spielen können (Baurecht, Gewerberecht, Polizeirecht) ${ }^{5}$. Folgende sieben Merkmale charakterisieren das Konzept:

- Der zu Grunde gelegte Regeltypus des Verwaltens ist die Hoheitsverwaltung. Allein das für diese Verwaltungstätigkeit typische öffentlich-rechtliche Handeln ist Gegenstand des Verwaltungsverfahrensgesetzes ( $§ 1$ Abs. 1).

- Das Gesetz ist in einem engeren Sinne entscheidungsorientiert. Es geht um rechtsförmige Entscheidungen, die in ihrer herausgehobenen Gestalt vom eigentlichen Verfahren abgesetzt und verselbständigt worden sind.

- Unter den Entscheidungen sind es wiederum die Einzelentscheidungen, die das Gesetz in den Mittelpunkt rückt (§ 9). Das erfasste Verwaltungshandeln ist Vollzugshandeln. Die administrative Normsetzung als Form einer zwischen dem parlamentarischen Gesetz und dem Einzelvollzug tretende Konkretisierung und Selbstprogrammierung hat in der Systematik des Gesetzes keinen Platz.

- Unter den Vollzugsentscheidungen dominieren die einseitigen Regelungen. Das Zentralinstitut ist der Verwaltungsakt. Öffentlich-rechtliche Verträge der Verwaltung finden zwar ebenfalls Anerkennung. Das Gesetz interessiert sich allerdings vor allem für (subordinationsrechtliche) Verträge in Situationen, in denen der Vertrag den Verwaltungsakt ersetzt ( $\$ 54$ S. 2).

- Das Gesetz geht von einer dienenden Funktion der verfahrensrechtlichen Regelungen gegenüber dem materiellen Recht aus (§ 46). Dahinter steht ein spezifisches Richtigkeitsverständnis von Entscheidungen, das der gerichtlichen Kontrolle die zentrale Position zuweist.

- Die kommunikativen Beziehungen zwischen Bürger und Verwaltung werden als Rechtsverhältnis mit festen Rollen definiert. Der Bürger verfolgt seine individuellen Rechte; die Verwaltung ist zwar rechtsgebunden, aber für die Definition des Gemeinwohls letztlich allein verantwortlich. Besonders deutlich zeigt sich das bei den Ausprägungen des Untersuchungsgrundsatzes (§ 24).

- Die Interessen der Bürger sind nach den Vorstellungen des Gesetzes vor allem auf die Abwehr belastender Eingriff und auf den Bestandserhalt einmal erlangter Vergünstigungen gerichtet. Rechtssicherheit, Bestandskraft und Vertrauensschutz spielen eine zentrale Rolle ( $\S 43,48,59)$. Der Vertrauensschutz war ein Schlüsselthema bei der Ausarbeitung des Gesetzes.

\subsubsection{Umfang und Art der Regelungen}

Das VwVfG erfüllt die Anforderungen an eine Kodifikation jedenfalls insoweit, als es klar gegliedert und nicht zu umfangreich ist. Sein Kern, das eigentliche Verfahrensrecht (Teil II), besteht aus ca. 25 Vorschriften. Dabei geht es keineswegs nur um allgemeine Grundsätze, sondern um ganz bestimmte Rechte und Pflichten sowie um einzelne feste Rechtsinstitute wie z.B. Fristen ( $\S 31,32$ VwVfG). Auf "soft law" ist verzichtet worden. Die Vorschriften sind Recht und müssen als Recht interpretiert und angewendet werden. Hilfreich ist es, dass in einem ersten Schritt das Recht, bzw. die Pflicht fixiert und in einem zweiten Schritt Ausnahmen genannt werden. Das erhöht die Anschaulich-

${ }^{5}$ Für die wichtigen Materien des Steuerrecht und des Sozialrecht gilt zwar nicht das VwVfG. Die entsprechenden „Teil-Kodifikationen“ (Abgabenordnung, Sozialgesetzbuch) enthalten aber weitgehen identisches Verfahrensrecht. 
keit. Die Formulierungen des Gesetzes schließen die Fortentwicklung durch Richterrecht nicht aus. Sie geben den Gerichten dazu aber einen greifbaren Text an die Hand, auf den die Argumentationen Bezug nehmen können.

(a) Nicht unbedingt notwendig erscheint es besondere Verfahren, wie z.B. über das Planfeststellungsverfahren, so ausführlich zu regeln, wie das VwVfG das in Teil V und Teil VII getan hat. Interessant für den Bürger ist von diesen Vorschriften vor allem die Regelung, welche Rechtsbehelfe („Beschwerde“, „Widerspruch“, gerichtliche „Klage“) gegen einen Verwaltungsakt eingelegt werden können. Dieses ist die Schnittstelle zwischen dem Verwaltungsverfahren und dem Verwaltungsprozess ${ }^{6}$. Das VwVfG sagt zu diesem wichtigen Thema nur wenig ( $\$ 80 \mathrm{VwVfG}$ - Kostentragung) und verweist im Übrigen auf die Verwaltungsgerichtsordnung. Das erscheint zwar akzeptabel, ist aber für den Bürger nicht optimal. Ein Verwaltungsverfahrensgesetz sollte (auch) darüber informieren, mit welchen Mitteln sich die Beteiligten gegen die von der Verwaltung getroffene Entscheidung wehren können und wie das zu geschehen hat (Form, Frist, Gegenstand).

(b) Richtigerweise hat das VwVfG Vorschriften über den Verwaltungsakt (Teil III) und den öffentlich-rechtlichen Vertrag (Teil IV) als „konnexe Materien“ einbezogen. Diese Vorschriften sind zwar eigentlich kein Verfahrensrecht im strengen Sinne. Aber sie betreffen das Ergebnis des Verfahrens und sie beantworten im Blick auf dieses Ergebnis wichtige Fragen, z.B. inwieweit das Ergebnis stabil ist („Rechtsicherheit“) und der Bürger sich darauf verlassen kann (,Vertrauensschutz").

Aus diesem Grunde bietet es sich an, auch das Verfahren der Verwaltungsvollstreckung einzubeziehen. Die Basis dieses Verfahrens sind die Ergebnisse, die das voraufgehende Verwaltungsverfahren abgeschlossen haben: eben der Verwaltungsakt oder der Vertrag. Es geht also um eine Form der Realisation der Verfahrensergebnisse, für die sich eine Einbeziehung in eine Kodifikation des Verfahrensrechts anbietet, ohne allerdings zwingend notwendig zu sein. In Deutschland ist die Verwaltungsvollstreckung in einem eigenen Gesetz geregelt.

(c) Vorschriften über den Datenschutz und über den freien Zugang zu Verwaltungsinformationen enthält das VwVfG nicht. Systematisch stellen sich diese Probleme auch nicht speziell für das Verwaltungsverfahren. Vielmehr gelten die entsprechenden Pflichten der Verwaltung unabhängig davon, ob ein Verwaltungsverfahren läuft. Sie sind in Deutschland in besonderen Gesetzen geregelt. Für das Verwaltungsverfahren selbst genügt eine Regelung, die die Verwaltung dazu verpflichtet, die Geheimnisse der Beteiligten, insbesondere die zum persönlichen Lebensbereich gehörenden Geheimnisse, nicht unbefugt zu offenbaren ( $\$ 30 \mathrm{VwVfG}$ ). Jüngere Kodifikationen beziehen diese Themen eines modernen Informationsveraltungsrechts allerdings ein. Wenn man eine zentrale Kodifikation schaffen will, die über die ,Verwaltungskultur" umfassend informiert, kann sich das empfehlen. Die Kodifikation wird dann jedoch erheblich umfangreicher und kann ihre Transparenz verlieren.

\subsection{Der US-amerikanische Administrative Procedure Act (APA) von 1946}

Auch die USA sind ein föderaler Staat. Anders als im deutschen System wird das Bundesrecht dort aber nicht von den Verwaltungen der Länder ("states"), sondern von

${ }^{6}$ Die deutsche Sprache ermöglicht es, genau zwischen (administrativem) „Verfahren“ und (judikativem) „Prozess“ zu unterscheiden. Entsprechend wird zwischen Verwaltungsverfahrensgesetz und Verwaltungsprozessgesetz (,Verwaltungsprozessordnung“) unterschieden. 
eigenen föderalen Behörden (“agencies”) vollzogen. Eine Besonderheit des amerikanischen Rechts ist es, dass viele (aber nicht alle!) dieser Behörden nur einer sehr begrenzten Kontrolle durch den Präsidenten der USA unterliegen und als "independent agencies" eigene Politik machen. Nach den einschlägigen Gesetzen, die die Aufgaben dieser Behörden oft nur sehr allgemein und unbestimmt festlegen, sollen sie das auch eigenständig tun. Dazu stehen ihnen legislative und judikative Instrumente zur Verfügung. Independent agencies werden in der Literatur als Vierte Gewalt ("forth branch") bezeichnet. Für das System der Gewaltenteilung, das die Verfassung der USA in den Art. I-III festgelegt hat, sind diese Behörden eine große Herausforderung (Schwartz, 1991).

IVor diesem Hintergrund ist 1946 der Administrative Procedure Act erlassen worden. Seine Vorbereitungen reichen in die Zeit des amerikanischen New Deal unter Präsident Roosevelt zurück. ,The key point in this interim period is the development of a "working compromise" between New Deal enthusiasts and those most critical of new regulatory institutions" (Breyer et al., 2011: 22). Der APA soll eine Art prozeduraler Kontrolle installieren, um die Unabhängigkeit der Behörden zu balancieren und die Unbestimmtheit der Gesetze durch eine breite Beteiligung von Interessierten Kreisen zu kompensieren. „Insgesamt liegt dem amerikanischen Recht die Überlegung zugrunde, dass sich die durch Verwaltungsentscheidungen von hoher Komplexität oder großem Konfliktpotential ausgelösten Spannungen am besten durch detailliert geregelte und gerichtlich kontrollierte Einflussmöglichkeiten für alle Interessierte lösen lassen“ (Erichsen et al., 2010). Das ist ein deutlich anderes Konzept als das Konzept des VwVfG. Stark vereinfachend kann man sagen: Der APA nimmt das politisch-administrative System insgesamt in den Blick, während das VwVfG vor allem das Verhältnis des einzelnen Bürger zur Verwaltung schützen will.

Beide Gesetze stimmen allerdings darin überein, dass sie die Verwaltung rechtlich ordnen wollen. Auch das amerikanische Verwaltungsrecht ist Recht, nicht nur ein politisches Programm. Es kann vor den Gerichten geltend gemacht werden. Die Gerichte, die in den USA eine starke Position haben, kontrollieren gerade Einhaltung des Verfahrensrechts sehr genau - genauer als in Deutschland, wo der Topos von der nur ,dienenden Funktion" des Verfahrens (vgl. oben unter A II 2) einen breiteren Anwendungsbereich hat (vgl. § $46 \mathrm{VwVfG})$.

\subsubsection{Umfang und Art der Regelungen}

Beide Kodifikationen, der APA ebenso wie das VwVfG, greifen auf die traditionellen Instrumente des prozeduralen Rechts zurück, vor allem den Gedanken der Anhörung („Hearing“). Aber beide haben eine unterschiedliche Perspektive. Während sich das VwVfG auf Einzelmaßnahmen der Verwaltung (Verwaltungsakte und Verträge) beschränkt, bezieht der APA die Normsetzung der Verwaltung ein. Verträge, die die Verwaltung mit Bürgern schließen, sind dagegen nicht Gegenstand des APA.

(a) Der APA beginnt mit einer langen Reihe von Definitionen (§ 552). Dadurch wird juristische Präzision gewährleistet. Dieser Vorteil geht allerdings auf Kosten der Verständlichkeit für den Laien. Dieser Eindruck setzt sich bei der Lektüre der folgenden Bestimmungen fort. Die Vorschriften sind außerordentlich lang; sie lesen sich kompliziert. Ihre Struktur ist systematisch exakt, aber (jedenfalls auf den ersten Blick) wenig transparent. Es ist ein Text für Experten, die sich mit herausgehobenen Fällen beschäf- 
tigen, während das VwVfG eher als ein Gesetz für die Alltagsarbeit der Behörden, auch derjenigen auf der lokalen Ebene, erscheint.

(b) Für die Regelung von Einzelfällen ("adjudication") stellt der APA ein förmliches Verfahren zur Verfügung ( $§ 554)$. Es läuft in der Art eines Gerichtsverfahrens ab. In seinem Zentrum steht ein formelles "hearing". In diesem können die Beteiligten ihre Argumente vorbringen können. Hier erfolgt auch die Beweisaufnahme, z.B. durch eine Vernehmung von Zeugen. Den Abschluss bildet ein zusammenfassender detaillierte Bericht ("record"). Dieser ist dann die Basis, auf der die Behörde ihre Entscheidung zu treffen hat ( $\$ 557)$. Über die informelle Regelung von Einzelfällen sagt der APA nicht. Sie läuft außerhalb der Kodifikation nach den richterrechtlich entwickelten Regeln der due process-clause.

(c) Für den Erlass von administrativen Normen ("rulemaking") stellt der APA mehrere Verfahrensarten zur Verfügung (Schwartz, 1991): In der Praxis besonders wichtig ist das sogenannte informal rulemaking ( $\$ 553)$. Es besteht aus drei Schritten: zunächst hat die Behörde öffentlich bekannt zu machen, dass sie den Erlass einer Norm beabsichtigt. Danach hat sie interessierten Personen Gelegenheit zu geben, zu dem Projekt ihre Ansichten und Informationen darzulegen. Das kann entweder schriftlich oder mündlich geschehen. Beim Erlass der vorgesehenen Norm muss sich die Behörde in einem genauen Bericht ("concise general statement") diesen Informationen auseinandersetzen. Dieses “notice and comment”-Verfahren ist ein ganz zentraler Bestandteil des amerikanischen Verwaltungsrechts, das in den Lehrbüchern breit behandelt wird (Breyer et al., 2011: 551-600).

(d) Besondere Bedeutung ist schon früh auch dem Informationsrecht beigemessen worden. Vorschriften über den Zugang zu Dokumenten waren zwar noch nicht im ursprünglichen APA von 1946 enthalten. Die durch solche Vorschriften angestrebte Transparenz der Verwaltung lag jedoch von Anfang an in der „Verfahrensphilosophie“. Durch den "Freedom of Information Act" sind 1966 entsprechende Vorschriften geschaffen und in den APA integriert worden (§ 552). Das amerikanische Verwaltungsrecht kann insofern durchaus als vorbildlich angesehen werden (Pierce et al., 2014). Zu kritisieren ist nur die außerordentlich komplizierte und schwer verständliche Art der Regelung.

Insgesamt aber kann der APA ebenso wie das VwVfG als eine gut durchdachte und für das Land repräsentative Kodifikation angesehen werden. Natürlich mussten beide Gesetze Kompromisse machen. Die Schaffung und Kodifizierung von Verwaltungsverfahrensrecht ist immer eine Gratwanderung zwischen zu vielen und zu wenigen Regeln, zwischen zu flexiblen und zu rigiden Vorschriften. Verfahrensrecht ist eine Frage des richtigen Maßes. Beide Gesetze haben diese Interessen abgewogen. Dabei zeigen sich unterschiedliche Präferenzen, die mit der unterschiedlichen politischen Kultur in den beiden Staaten zu tun haben. Aber auf diese Weise sind ausgewogene Modelle entstanden.

\subsection{Der ReNEUAL-Musterentwurf für ein EU-Verwaltungsverfahrensrecht von 2014}

Dieser Entwurf einer Kodifikation ist von einer Arbeitsgruppe erarbeitet worden, in der sich elf Wissenschaftler aus unterschiedlichen europäischen Ländern aus eigener Initiative zusammengefunden haben (Schneider et al., 2015). Er ist zunächst ein- 
mal ein Projekt der Wissenschaft. Er hat die unterschiedlichen Verfahrensregelungen des EU-Rechts und der Mitgliedstaaten zusammengetragen, sorgfältig analysiert und daraus einen innovativen Kodifikationsvorschlag entwickelt. Das Gesetz soll (jedenfalls zunächst) nur für die eigene Verwaltung der EU, nicht aber für die Verwaltungen der Mitgliedstaaten gelten.

\subsubsection{Das Konzept}

Der ReNEUAL-Entwurf legt das Konzept einer umfassenden Kodifikation zugrunde. Er ist in sechs Bücher gegliedert. Drei Bücher behandeln das Verwaltungsverfahren der wichtigsten administrativen Handlungsformen: die Normsetzung der Exekutive (Buch II), die Einzelfallentscheidungen (Buch III) und die Verträge der Verwaltung (Buch IV). Der Entwurf greift damit über das deutsche VwVfG und über den amerikanischen APA hinaus. Für die Normsetzung wird ein dem APA ähnliches “notice and comment"-Verfahren vorgeschlagen. Für das Verfahren zum Erlass von Einzelakten bezieht sich der Entwurf mehrfach auf das deutsche VwVfG. Wie dieses beschäftigt er sich auch mit der das Verfahren abschließenden Entscheidung (,Verwaltungsakt") und der Frage ihrer späteren Abänderbarkeit.

Besonders innovativ ist es, in einem besonderen Buch das Informationsmanagement der Verwaltung zu regeln (Buch VI). Dieses Thema ist für die Verwaltung in der EU besonders wichtig, weil sie zu großen Teilen auf dem Austausch von Informationen (vertikal) zwischen den Verwaltungen der Mitgliedstaaten und der EU-Verwaltung und (horizontal) zwischen den Verwaltungen der Mitgliedstaaten untereinander beruht. Hier spielen Datenbanken und Informationsnetzwerke eine besondere Rolle. Die Fragen, inwieweit der Informationsaustausch verlangt werden kann und welche rechtlichen Grenzen dabei einzuhalten sind, müssen für den ,Europäischen Verwaltungsverbund" exakt geregelt werden". Diese Regelungen des Entwurfs beziehen notwendig auch die Verwaltungen der Mitgliedstaaten ein. Indem das Informationsthema hier kreativ aufgegriffen wird, indiziert der Entwurf die Notwendigkeit, über eine weitere, eine neue „dritte“ Generation von Verwaltungsverfahren nachzudenken (Barnes, 2010: 336).

Für einen einzelnen Staat, der nicht Mitglied eines Verwaltungsverbundes ist, ist das Buch VI des Entwurfs nicht direkt einschlägig. Es ist jedoch interessant, weil es die Bedeutsamkeit des Informationsrechts der Verwaltung unterstreicht. Einfache Regeln über die Amtshilfe zwischen Behörden, in denen auch die entsprechenden Fragen des Datenschutzes geregelt sind, sollten in einem modernen Verwaltungsverfahrensgesetz enthalten sein. Die Vorschriften des VwVfG (§§ 4-8) genügen diesen Anforderungen an eine Integration datenschutzrechtlicher Bestimmungen in das Verfahrensrecht der Amtshilfe nicht (mehr). Der ReNEUAL-Entwurf enthält dazu wesentlich differenziertere Regelungen, die auch ein Benachrichtigung der von einem Informationsaustausch betroffenen Bürger vorsehen (Buch V).

\subsubsection{Die Realisierung des Projekts}

Der Entwurf ist wie gesagt zunächst einmal ein Projekt der Wissenschaft. Ob und wie er in verbindliche Rechtsakte de EU umgesetzt wird, ist zurzeit noch nicht vorhersehbar.

\footnotetext{
${ }^{7}$ Zum Gedanken des „Europäischen Verwaltungsverbundes“ (Schmidt-Aßmann, 2012b).
} 
Dass es erhebliche politische Widerstände geben wird, ist sehr wahrscheinlich. Immerhin genießt das Projekt aber die Unterstützung des Europäischen Parlaments. Entscheidend wird es sein, wie sich die Kommission der EU zu dem Entwurf stellt. Sie hat das Initiativrecht.

Der Entwurf folgt dem Modell einer integrierenden Kodifikation: Die sechs Bücher sind gleichzeitig entworfen worden und im Detail aufeinander abgestimmt. Das bedeutet aber nicht notwendig, dass alle Bücher gleichzeitig in Kraft gesetzt werden müssen. Auch eine sukzessive Kodifizierung ist denkbar. Nach einem solchen Modell sind etwa die Niederlande seit 1994 vorgegangen. Auch für den ReNEUAL-Entwurf wird in der Literatur darüber nachgedacht, schrittweise vorzugehen. Dann würde es sich anbieten, mit Buch III zu beginnen. Das Verfahren zum Erlass von Einzelakten ist der klassische Typ eines Verwaltungsverfahrens. Seine wichtigsten Bestandteile sind durch den Rechtsvergleich gut belegt. Die Verfasser des ReNEUAL-Entwurfs konnten hier auf sicherem Boden arbeiten und sind zu Vorschlägen gelangt, die in ihren Formulierungen und in ihrem Inhalt durchgängig überzeugen.

Insgesamt unterstreicht der ReNEUAL-Entwurf, dass die grundlegenden Regelungen eines modernen Allgemeinen Verwaltungsrechts heute europaweit und darüber hinaus „Kodifikationsreife“ erlangt haben.

\section{Bibliographie:}

1. Administrative Law and Process / R. Pierce, S. Shapiro, P. Verkuil. $6^{\text {th }}$ ed. New York : Foundation Press, 2014. 493 p.

2. Administrative Law and Regulatory Policy: Problems Text, and Cases / S. Breyer, R. Stewart, C. Sunstein, A. Vermeule, M. Herz. $7^{\text {th }}$ ed. New York, 2011. 700 p.

3. Allgemeines Verwaltungsrecht / H.-U. Erichsen, D. Ehlers (Hrsg.). 14. Auflage. Berlin ; New York : De Gruyter, 2010. 1066 S.

4. Barnes J. Towards a third generation of administrative procedure. Comparative Administrative Law / S. Rose-Ackerman, P.L. Lindseth (eds.). Cheltenham ; Northampton, 2010. P. 336-380.

5. Battini S., Materella B., Sandulli A. Il procedimento. Diritto Amministrativo Comparato / G. Napolitano (ed.). Milano : Giuffrè, 2007. P. 107-174.

6. Bell J.S. Comparative Administrative Law. The Oxford Handbook of Comparative Law / M. Reimann, R. Zimmermann (eds.). Oxford : Oxford University Press, 2006. P. 1259-1277.

7. Beschluss vom 10.05.1977 / Bundesverfassungsgericht. Amtlichen Entscheidungssammlung. 1977. Bd. 45. S. 297-335.

8. Beschluss vom 20.12.1979 / Bundesverfassungsgericht. Amtlichen Entscheidungssammlung. 1979. Bd. 53. S. 30-75.

9. Comparative Administrative Law / S. Rose-Ackerman, P.L. Lindseth (eds.). Cheltenham ; Northampton, 2010. 688 p.

10. Craig P. A general law on administrative procedure, legislative competence and judicial competence. European Public Law. 2013. Vol. 19. Issue 3. P. 503-524.

11. Craig P. Administrative Law. $7^{\text {th }}$ ed. London : Sweet \& Maxwell, 2012. 1102 p.

12. Deppe J., Pudelka J. General Administrative Law in Central Asian States - a brief overview of the current state. URL: http://ruleoflaw.en/wp-content/uploads/2014/01/130708-PudelkaDeppe-study_r.pdf.

13. Entscheidungen vom 31.10.1990 / Bundesverfassungsgericht. Amtlichen Entscheidungssammlung. 1990. Bd. 83. S. 37-80. 
14. Fromont M. Droit administratif des États européens. Paris : Presses Universitaires de France, 2006. 362 p.

15. Jaeckel L. Gefahrenabwehrrecht und Risikodogmatik. Tübingen, 2010. 379 S.

16. Kahl W. Das Verwaltungsverfahrensgesetz zwischen Kodifikationsidee und Sonderrechtsentwicklungen. Verwaltungsverfahren und Verwaltungsverfahrensgesetz / W. Hoffmann-Riem, E. Schmidt-Aßmann (Hrsg.). Baden-Baden, 2002. S. 67-90.

17. Pitschas R. Verwaltungsverantwortung und Verwaltungsverfahren. München, 1990. $805 \mathrm{~S}$.

18. Pünder H. Administrative Procedure - Mere Facilitator of Material Law versus Cooperative Realization of Common Welfare (Chapter 13). Debates in German Public Law / H. Pünder, C. Waldhoff (eds.). Oxford, 2014. S. 239-260.

19. Quabeck C. Dienende Funktion des Verwaltungsverfahrens und Prozeduralisierung. Tübingen, 2010. $323 \mathrm{~S}$.

20. ReNEUAL - Musterentwurf für ein EU-Verwaltungsverfahrensrecht / J.-P. Schneider, H.C.H. Hofmann, J. Ziller (Hrsg.). München, 2015. 214 S.

21. Röhl H.Ch. Ausgewählte Verwaltungsverfahren (§ 30). Grundlagen des Verwaltungsrechts $(G V w R)$ / W. Hoffmann-Riem, E. Schmidt-Aßmann, A. Voßkuhle (Hrsg.). 2. Auflage. München, 2012. Bd. 2. S. 731-798.

22. Rose-Ackerman S., Perroud T. Policy Making and Public Law in France: Public Participation, Agency Independence and Impact Assessment. Columbia Journal of European Law. 2013. Vol. 19. P. 225-312.

23. Schmidt-Aßmann E. Das Allgemeine Verwaltungsrecht als Ordnungsidee. 2. Auflage. Berlin, 2004. $470 \mathrm{~S}$.

24. Schmidt-Aßmann E. Der Verfahrensgedanke im deutschen und europäischen Verwaltungsrecht (§ 27). Grundlagen des Verwaltungsrechts $(G V w R) /$ W. Hoffmann-Riem, E. SchmidtAßmann, A. Voßkuhle (Hrsg.). 2. Auflage. München, 2012. Bd. 2. S. 495-556.

25. Schmidt-Aßmann E. Grundrechte als Organisations- und Verfahrensgarantien (§ 45). Handbuch der Grundrechte (HGR) / D. Merten, H.-J. Papier (Hrsg.). Heidelberg, 2006. Bd. 2. S. 993-1030.

26. Schmidt-Aßmann T. Verfassungsprinzipien für den Europäischen Verwaltungsverbund (§ 5). Grundlagen des Verwaltungsrechts $(G V w R)$ / W. Hoffmann-Riem, E. Schmidt-Aßmann, A. Voßkuhle (Hrsg.). 2. Auflage. München, 2012. Bd. 1. S. 261-340.

27. Schneider J.-P. Strukturen und Typen von Verwaltungsverfahren (§ 28). Grundlagen des Verwaltungsrechts (GVwR) / W. Hoffmann-Riem, E. Schmidt-Aßmann, A. Voßkuhle (Hrsg.). 2. Auflage. München, 2012. Bd. 2. S. 557-662.

28. Schwartz B. Administrative Law. $3^{\text {rd }}$ ed. Boston, 1991. 816 p.

29. Trute H.-H. Die demokratische Legitimation der Verwaltung (§ 6). Grundlagen des Verwaltungsrechts $(G V w R)$ / W. Hoffmann-Riem, E. Schmidt-Aßmann, A. Voßkuhle (Hrsg.). 2. Auflage. München, 2012. Bd. 1. S. 341-436.

30. Verwaltungsrecht in Europa. Bd. 1 (England, Spanien, Niederlande) / J.-P. Schneider (Hrsg.). Göttingen, 2007. $471 \mathrm{~S}$.

31. Verwaltungsrecht in Europa. Bd. 2 (Frankreich, Tschechien, Polen) / J.-P. Schneider (Hrsg.). Göttingen, 2009. 494 S.

32. Verwaltungsverfahrensgesetzes (VwVfG) von 1973 / Deutschen Bundestag. Bundestagsdrucksache. 1973. № 7/910. S. 27-130.

33. Wade W., Forsyth C. Administrative Law. $11^{\text {th }}$ ed. Oxford, 2014. 958 p.

34. Wollenschläger B. Wissensgenerierung im Verfahren. Tübingen, 2009. 259 S. 


\section{References:}

1. Pierce, R., Shapiro, S., Verkuil, P. (2014). Administrative Law and Process. $6^{\text {th }}$ ed. New York: Foundation Press [in English].

2. Breyer, S., Stewart, R., Sunstein, C., Vermeule, A., Herz, M. (2011). Administrative Law and Regulatory Policy: Problems Text, and Cases. $7^{\text {th }}$ ed. New York [in English].

3. Erichsen, H.-U., Ehlers, D. (eds.) (2010). Allgemeines Verwaltungsrecht [General administrative law]. 14th ed. Berlin; New York: De Gruyter [in German].

4. Barnes, J. (2010). Towards a third generation of administrative procedure. Rose-Ackerman, S., Lindseth, P.L. (eds.). Comparative Administrative Law. Cheltenham; Northampton, pp. 336-380 [in English].

5. Battini, S., Materella, B., Sandulli, A. (2007). Il procedimento [The process]. Napolitano, G. (ed.). Diritto Amministrativo Comparato. Milano: Giuffrè, pp. 107-174 [in Italian].

6. Bell, J.S. (2006). Comparative Administrative Law. Reimann, M., Zimmermann, R. (eds.). The Oxford Handbook of Comparative Law. Oxford: Oxford University Press, pp. 1259-1277 [in English].

7. Federal Constitutional Court (1977). Beschluss vom 10.05.1977 [Decision of May 10, 1977]. Amtlichen Entscheidungssammlung, issue 45, pp. 297-335 [in German].

8. Federal Constitutional Court (1979). Beschluss vom 20.12.1979 [Decision of December 20, 1979]. Amtlichen Entscheidungssammlung, issue 53, pp. 30-75 [in German].

9. Rose-Ackerman, S., Lindseth, P.L. (eds.) (2010). Comparative Administrative Law. Cheltenham; Northampton [in English].

10. Craig, P. (2013). A general law on administrative procedure, legislative competence and judicial competence. European Public Law, vol. 19, issue 3, pp. 503-524 [in English].

11. Craig, P. (2012). Administrative Law. $7^{\text {th }}$ ed. London: Sweet \& Maxwell [in English].

12. Deppe, J., Pudelka, J. (2014). General Administrative Law in Central Asian States - a brief overview of the current state. Retrieved from: http://ruleoflaw.en/wp-content/ uploads/2014/01/130708-Pudelka-Deppe-study_r.pdf [in English].

13. Federal Constitutional Court (1990). Entscheidungen vom 31.10.1990 [Decisions of October 31, 1990]. Amtlichen Entscheidungssammlung, issue 83, pp. 37-80 [in German].

14. Fromont, M. (2006). Droit administratif des États européens [Administrative law of European states]. Paris: Presses Universitaires de France [in French].

15. Jaeckel, L. (2010). Gefahrenabwehrrecht und Risikodogmatik [Defense law and risk dogmatics]. Tübingen [in German].

16. Kahl, W. (2002). Das Verwaltungsverfahrensgesetz zwischen Kodifikationsidee und Sonderrechtsentwicklungen [The Administrative Procedures Act between the idea of codification and developments in special law]. Hoffmann-Riem, W., Schmidt-Aßmann, E. (eds.). Verwaltungsverfahren und Verwaltungsverfahrensgesetz. Baden-Baden, pp. 67-90 [in German].

17. Pitschas, R. (1990). Verwaltungsverantwortung und Verwaltungsverfahren [Administrative responsibility and administrative procedures]. München [in German].

18. Pünder, H. (2014). Administrative Procedure - Mere Facilitator of Material Law versus Cooperative Realization of Common Welfare (Chapter 13). Pünder, H., Waldhoff, C. (eds.). Debates in German Public Law. Oxford, pp. 239-260 [in English].

19. Quabeck, C. (2010). Dienende Funktion des Verwaltungsverfahrens und Prozeduralisierung [Serving function of the administrative procedure and proceduralization]. Tübingen [in German].

20. Schneider, J.-P., Hofmann, H.C.H., Ziller, J. (eds.) (2015). ReNEUAL - Musterentwurf für ein EU-Verwaltungsverfahrensrecht [ReNEUAL - Model for an EU administrative procedural law]. München [in German]. 
21. Röhl, H.Ch. (2012). Ausgewählte Verwaltungsverfahren (§ 30) [Selected administrative procedures (§ 30)]. Hoffmann-Riem, W., Schmidt-Aßmann, E., Voßkuhle, A. (eds.). Grundlagen des Verwaltungsrechts (GVwR). 2nd ed. München, vol. 2, pp. 731-798 [in German].

22. Rose-Ackerman, S., Perroud, T. (2013). Policy Making and Public Law in France: Public Participation, Agency Independence and Impact Assessment. Columbia Journal of European Law, vol. 19, pp. 225-312 [in English].

23. Schmidt-Aßmann, E. (2004). Das Allgemeine Verwaltungsrecht als Ordnungsidee [General administrative law as an idea of order]. 2nd ed. Berlin [in German].

24. Schmidt-Aßmann, E. (2012a). Der Verfahrensgedanke im deutschen und europäischen Verwaltungsrecht (§ 27) [The procedural idea in German and European administrative law (§ 27)]. Hoffmann-Riem, W., Schmidt-Aßmann, E., Voßkuhle, A. (eds.). Grundlagen des Verwaltungsrechts $(G V w R)$. 2nd ed. München, vol. 2, pp. 495-556 [in German].

25. Schmidt-Aßmann, E. (2006). Grundrechte als Organisations- und Verfahrensgarantien (§ 45) [Fundamental rights as organizational and procedural guarantees (§ 45)]. Merten, D., Papier, H.-J. (eds.). Handbuch der Grundrechte (HGR). Heidelberg, vol. 2, pp. 993-1030 [in German].

26. Schmidt-Aßmann, T. (2012b). Verfassungsprinzipien für den Europäischen Verwaltungsverbund $(\S 5)$ [Constitutional principles for the European administrative group ( $(5)$ ]. Hoffmann-Riem, W., Schmidt-Aßmann, E., Voßkuhle, A. (eds.). Grundlagen des Verwaltungsrechts $(G V w R)$. 2nd ed. München, vol. 1, pp. 261-340 [in German].

27. Schneider, J.-P. (2012). Strukturen und Typen von Verwaltungsverfahren (§ 28) [Structures and types of administrative procedures (§ 28)]. Hoffmann-Riem, W., Schmidt-Aßmann, E., Voßkuhle, A. (eds.). Grundlagen des Verwaltungsrechts (GVwR). 2nd ed. München, vol. 2, pp. 557-662 [in German].

28. Schwartz, B. (1991). Administrative Law. $3^{\text {rd }}$ ed. Boston [in English].

29. Trute, H.-H. (2012). Die demokratische Legitimation der Verwaltung (§ 6) [The democratic legitimacy of the administration (§ 6)]. Hoffmann-Riem, W., Schmidt-Aßmann, E., Voßkuhle, A. (eds.). Grundlagen des Verwaltungsrechts (GVwR). 2nd ed. München, vol. 1, pp. 341-436 [in German].

30. Schneider, J.-P. (ed.) (2007). Verwaltungsrecht in Europa. Bd. 1 (England, Spanien, Niederlande) [Administrative law in Europe. Vol. 1 (England, Spain, Netherlands)]. Göttingen [in German].

31. Schneider, J.-P. (ed.) (2009). Verwaltungsrecht in Europa. Bd. 2 (Frankreich, Tschechien, Polen) [Administrative law in Europe. Vol. 2 (France, Czech Republic, Poland)]. Göttingen [in German].

32. German Bundestag (1973). Verwaltungsverfahrensgesetzes (VwVfG) von 1973 [Administrative Procedure Act (VwVfG) of 1973]. Bundestagsdrucksache, no. 7/910, pp. 27-130 [in German].

33. Wade, W., Forsyth, C. (2014). Administrative Law. $11^{\text {th }}$ ed. Oxford [in English].

34. Wollenschläger, B. (2009). Wissensgenerierung im Verfahren [Knowledge generation in the process]. Tübingen [in German]. 


\title{
CODIFICATION OF ADMINISTRATIVE PROCEDURAL LAW: TRADITIONS AND MODELS
}

\author{
CV Professor Dr. Dr. h.c. mult. \\ Eberhard Schmidt-Aßmann, \\ Professor Emeritus of Public Law \\ of the University of Heidelberg, Germany \\ e.schmidt-assmann@t-online.de
}

The contribution explains the underlying rationale, objectives and constitutional framework for administrative procedural law. Furthermore, the arguments for and against comprehensive codification of administrative procedural rules, as well as the most significant elements of administrative procedural law, are discussed. Lastly, possible models for codification are presented, namely the German Law on Administrative Procedure, the US Administrative Procedure Act and the ReNEUAL Model Rules on EU Administrative Procedure.

Key words: administrative law, good governance, rule of law, administrative procedural rules, codification, models, German Law on Administrative Procedure, US Administrative Procedure Act, ReNEUAL Model Rules on EU Administrative Procedure. 